\title{
Characteristics of Callers to the Domestic Abuse Helpline for Men
}

\author{
Denise A. Hines · Jan Brown • Edward Dunning
}

Published online: 15 August 2007

(C) Springer Science + Business Media, LLC 2007

In the online and print publications of "Characteristics of Callers to the Domestic Abuse Helpline for Men" (DOI: 10.1007/s10896-006-9052-0) the Acknowledgement as well as the address listed under the last author were incorrect.

The correct address is as follows:

E. Dunning

Family Interventions Project, 4300 Auburn Blvd., Suite 208,

Sacramento, CA 95841, USA

And the correct Acknowledgment is as follows:

In addition to thanking the members of the Family Research Laboratory for their comments on a previous draft of this article, the authors would also like to thank Mark Rosenthal for his help in preparing the data for analysis.

The online version of the original article can be found at http://dx.doi. org/10.1007/s10896-006-9052-0.

D. A. Hines $(\bowtie)$

Department of Criminal Justice,

University of Massachusetts-Lowell,

870 Broadway St.,

Lowell, MA 01801, USA

e-mail: Denise_Hines@uml.edu

J. Brown

Domestic Abuse Helpline for Men and Women,

P.O. Box 252, Harmony, ME 04942, USA

E. Dunning

Family Interventions Project,

4300 Arbors Blvd., Suite 208,

Sacramento, CA 95841, USA 